\title{
Video-assisted Thoracic Surgery for Congenital Diaphragmatic Hernia Compared With Conventional Open Surgery in Infants
}

\section{Shengliang Zhao}

Children's Hospital of Chongqing Medical University

\section{Zhengxia Pan}

Children's Hospital of Chongqing Medical University

\section{Yonggang $\mathrm{Li}$}

Children's Hospital of Chongqing Medical University

\section{Yong An}

Children's Hospital of Chongqing Medical University

\section{Xin Jin}

Children's Hospital of Chongqing Medical University chun wu ( $\square$ wuchun0312@sina.com )

Children's Hospital of Chongqing Medical University

\section{Research Article}

Keywords: Congenital diaphragmatic hernia, Minimally invasive surgery, Thoracoscopic, Infants

Posted Date: March 18th, 2021

DOl: https://doi.org/10.21203/rs.3.rs-310228/v1

License: (9) (i) This work is licensed under a Creative Commons Attribution 4.0 International License. Read Full License 


\section{Abstract}

\section{Background}

This study aimed to evaluate the effectiveness of video-assisted thoracic surgery for the treatment of congenital diaphragmatic hernia $(\mathrm{CDH})$ in a larger series compared with conventional open surgery. Additionally, we summarized the experience of thoracoscopic surgery in the treatment of CDH in infants.

\section{Methods}

We retrospectively analysed the clinical data of 109 children with $\mathrm{CDH}$ who underwent surgical treatment at the Department of Cardiothoracic Surgery of Children's Hospital of Chongqing Medical University from January 2010 to January 2019. According to the surgical method, the children were divided into an open group (62 cases) and a thoracoscopy group (47 cases). We compared the operation time, intraoperative blood loss, postoperative mechanical ventilation time, postoperative hospital stay, postoperative CCU admission time and other surgical indicators as well as the recurrence rate, mortality rate and complication rate of the two groups of children.

\section{Results}

The index data on the operation time, intraoperative blood loss, postoperative mechanical ventilation time, postoperative hospital stay and postoperative CCU admission time were better in the thoracoscopy group than in the open group. The difference between the two groups was statistically significant $(P<0.05)$. Compared with the number of incision infections, pulmonary infections, atelectasis, pleural effusion and chylothorax between the two groups, the number of children in the open group was greater than that in the thoracoscopy group, and the total postoperative complication rate (51.61\%) was higher than that in the thoracoscopy group (44.68\%). The recurrence rate of the thoracoscopy group $(8.51 \%)$ was higher than that of the open group (3.23\%). In the open group, 2 patients died of respiratory distress after surgery, and no patients died in the thoracoscopy group.

\section{Conclusions}

Thoracoscopic surgery and open surgery can effectively treat $\mathrm{CDH}$. Open surgery has advantages in patients with unstable haemodynamics, large diaphragm defects and abdominal malformations. Compared with conventional open surgery, thoracoscopy has the advantages of shorter operation time, less trauma, faster recovery and fewer complications, but there is a risk of recurrence. The choice of surgical method should be determined by the characteristics of diaphragmatic lesions and the experience of the clinician.

\section{Background}

Congenital diaphragmatic hernia $(\mathrm{CDH})$ is due to developmental disorders or dysplasia of the diaphragm. The contents of the abdominal cavity herniate into the thoracic cavity and compress the lungs and heart, 
which can lead to pulmonary dysplasia and pulmonary hypertension. The incidence of CDH is approximately 1:2500-1:5000 [1], and the fatality rate of $\mathrm{CDH}$ is as high as $40 \% \sim 60 \%$ [2-3]. The current treatment still requires surgical repair of the diaphragm defect, including open surgery and minimally invasive surgery. Open surgery generally involves traditional laparotomy or thoracotomy, and minimally invasive surgery is divided into laparoscopic and thoracoscopic surgery. Related studies have suggested that the operation time, hospitalization time, postoperative mechanical ventilation time and postoperative analgesic use of children after minimally invasive surgery are better than those after open surgery but that it may cause hypercapnia and hypoxia. Additionally, the recurrence rate after minimally invasive surgery is also higher [4]. However, comparative studies between minimally invasive surgery and open surgery have not been conducted extensively due to wide discrepancies in the severity of pulmonary hypoplasia and pulmonary hypertension between patients as well as different management protocols, resulting in the indications for minimally invasive surgery varying from centre to centre. We selected 109 children with $\mathrm{CDH}$ as the research subjects to observe the comparison of thoracoscopic diaphragmatic hernia repair with traditional open surgery to provide a reference basis for clinical decision-making regarding $\mathrm{CDH}$ surgery.

\section{Methods}

We retrospectively analysed the clinical data of 109 children with $\mathrm{CDH}$ admitted to the Department of Cardiothoracic Surgery, Children's Hospital of Chongqing Medical University from January 2010 to January 2019. The Medical Research Ethics Committee of Children's Hospital Affiliated to Chongqing Medical University approved the study, and this study obtained written informed consent from the families of all children. The study protocol is performed in accordance with the relevant guidelines.

The inclusion criteria were as follows: (1) children whose abdominal viscera (e.g., stomach, intestine, liver and kidney) herniated into the thoracic cavity by chest X-ray, gastrointestinal angiography or chest CT examination; (2) children and their family members signed the agreement voluntarily and agreed to accept the operation; and (3) children who were diagnosed with diaphragm defects during the operation. The exclusion criteria were as follows: (1) patients with other serious congenital malformations (digestive tract malformations, heart malformations and lung malformation) who underwent related deformity correction surgery at the same time; (2) patients combined with chromosomal genetic diseases; (3) children with congenital diaphragmatic eventration, acquired diaphragmatic hernia, traumatic diaphragmatic hernia, oesophageal hiatal hernia and parasternal hernia; and (4) children without surgery.

For the open surgery group, the right $\mathrm{CDH}$ was treated by a transthoracic approach, and the left $\mathrm{CDH}$ was treated through an abdominal approach. Through the thoracoabdominal approach, we removed the weak diaphragm and used intermittent nonabsorbable sutures to ensure that the cut diaphragm had a shingled shape to strengthen the weak area of the diaphragm. We once again sutured the ventral muscle layer of the diaphragm with intermittent mattresses and removed the hernia sac if it was combined. For the thoracoscopic group, a $5 \mathrm{~mm}$ trocar was placed on the lower edge of the scapula tip using the three-hole method, and two operation holes were made in the fourth intercostal space on both sides of the trocar. 
The thoracic cavity pressurizes the abdominal organs into the abdominal cavity and fully exposes the diaphragm defect to observe the size of the diaphragm defect. If there was a hernia sac in the child, it was pushed into the abdominal cavity, and a purse-string suture with a needled suture was used to repair the diaphragmatic defect, close the $\mathrm{CDH}$, restabilize and suture the edge of the defect with barbed sutures. In addition, a chest drainage tube was placed after surgery.

We observed the operation time, intraoperative blood loss, postoperative mechanical ventilation time, postoperative hospital stay, postoperative CCU admission time and other surgical indicators as well as the recurrence rate, mortality rate and complication rate of the two groups of children.

\section{Results}

This study included 109 children with $\mathrm{CDH}$. According to different surgical methods, 47 children who underwent thoracoscopic diaphragmatic hernia repair were included in the thoracoscopy group, and 62 children who underwent traditional open surgery were included in the open group. In the open group, 56 patients underwent transabdominal diaphragmatic hernia repair, and 6 patients underwent transthoracic hernia repair. There were 47 cases in the thoracoscopy group, including 25 males and 22 females who were aged $1 \mathrm{~h}$ to 1 year and 2 months (average of 9.05 \pm 3.97 months) and weighed $2.48-11.2 \mathrm{~kg}$ (average of $6.55 \pm 2.44 \mathrm{~kg}$ ). With regard to the location of the disease, 38 cases on the left side, and 9 cases on the right side. There were 62 patients in the open group, including 36 males and 26 males who were aged $2 \mathrm{~h}$ to 2 years (average of $8.83 \pm 6.11$ months) and weighed $2.51-14.2 \mathrm{~kg}$ (average of $7.04 \pm 3.73 \mathrm{~kg}$ ). With regard to the location of the disease, 51 cases were on the left side, and 11 cases were on the right side. In the open group, 10 cases were complicated with other malformations, including 3 cases of cryptorchidism, 2 cases of pectus excavatum and 5 cases of right heart shift. In the thoracoscopic group, 15 cases were complicated with other malformations, including 3 cases of cryptorchidism, 4 cases of pectus excavatum, 3 cases of right heart shift and 5 cases of congenital intestinal malrotation. There was no significant difference in sex, age, weight, location of disease or other preoperative data between the two groups $(P>0.05)$, indicating that the two groups were comparable (Table 1). All children in the open group completed the operation successfully, and 3 cases in the thoracoscopy group were converted to open surgery due to severe thoracic adhesions and abdominal organs obstructing the surgical field. During the operation, the orifice diameter of the $\mathrm{CDH}$ was 2-12 cm, and the average area of the diaphragmatic muscle defect was $18.12 \pm 6.43 \mathrm{~cm}^{2}$. The 89 cases with left $\mathrm{CDH}$ mainly herniated into the following organs: small intestine, stomach, colon, mesenteric and spleen. The 20 cases of right $\mathrm{CDH}$ mainly herniated into the right lobe of the liver, small intestine and colon.

We analysed the data of the relevant surgical indicators in the two groups. The index data on the operation time, intraoperative blood loss, postoperative mechanical ventilation time, postoperative hospital stay and postoperative CCU admission time were better in the thoracoscopy group than in the open group. The difference between the two groups was statistically significant $(p<0.05)$ (Table 2$)$. Compared with the number of incision infections, pulmonary infections, atelectasis, pleural effusion and chylothorax between the two groups, the number of children in the open group was greater than that in 
the thoracoscopy group, and the total postoperative complication rate $(45.16 \%)$ was higher than that in the thoracoscopy group (36.17\%). Both groups of children were followed up for 1 month to 9 years after surgery. Three patients in the thoracoscopy group relapsed, and 2 patients in the open group relapsed. The recurrence rate and mortality of children in the thoracoscopy group were not significantly different from those in the open group $(P>0.05)$ (Table 3$)$. Two cases in the open group died after surgery, and the causes of death were respiratory failure, pulmonary dysplasia and severe pulmonary hypertension. The rest of the children were followed up for growth and breathing in outpatient clinics or by telephone. After discharge from the hospital, 13 children were lost to long-term follow-up, and 96 patients were followed up for 1 month to 9 years. All the children recovered well without death or serious complications. Postoperative chest radiograph and gastrointestinal angiography showed that the abdominal organs were in normal positions (Figure 1).

\section{Discussion}

$\mathrm{CDH}$ is a congenital disease that causes a defect in the diaphragm due to abnormal embryonic development, causing abdominal organs to herniate into the thoracic cavity and causing a series of pathophysiological changes. Generally, $\mathrm{CDH}$ occurs more often on the left side than the right side with the incidence ratio on the left and right sides being approximately $6: 1$, and $\mathrm{CDH}$ is rare on both sides with an incidence rate of approximately $2 \%$ [5]. In this study, the number of children with $\mathrm{CDH}$ on the left side (81.65\%) was far greater than that of children with $\mathrm{CDH}$ on the right side (18.35\%), which was consistent with literature reports. The incidence of $\mathrm{CDH}$ combined with other malformations is $30 \%-70 \%$, including cardiovascular formations (27.5\%), genitourinary system malformations (17.7\%), skeletal muscle system malformations (15.7\%) and central nervous system malformations (9.8\%) [6]. In this group, congenital pulmonary dysplasia $(53,48.62 \%)$ and congenital heart disease $(45,41.28 \%)$ were the main malformations in this study. Other combined malformations included cryptorchidism, pectus excavatum, right shifted heart and congenital intestinal malrotation, which was consistent with literature reports.

The traditional treatment for $\mathrm{CDH}$ is transthoracic or transabdominal diaphragmatic hernia repair. In the open group, 56 patients underwent transabdominal diaphragmatic hernia repair, and 6 patients underwent transthoracic diaphragmatic hernia repair. We believe that transabdominal surgery has the following advantages: easy to reset abdominal organs; easy to suture the edge of the diaphragm; and easy to deal with abdominal deformities, such as intestinal rotation malrotation. Therefore, transabdominal surgery is recommended for left-side diaphragmatic hernias. Transthoracic surgery is used for right-side diaphragmatic hernia due to liver obstruction because the diaphragm is clearly exposed during transthoracic surgery. Additionally, transthoracic surgery addresses thoracic adhesions and other combined chest deformities. However, in the open group, 5 children with right CDH without thoracic malformations were treated with transabdominal diaphragmatic hernia repair, which also achieved satisfactory clinical results. Therefore, we believe that the choice of approach is mainly based on the characteristics of the patient's $\mathrm{CDH}$ lesions and which approach the surgeon is more familiar with. In this study, 47 patients had stable haemodynamics, no severe pulmonary dysplasia and pulmonary hypertension. In addition, thoracoscopic diaphragmatic hernia repair was used. A purse-string suture with 
a needled suture was used to repair the diaphragmatic defect, close the diaphragmatic hernia, restabilize and suture the edge of the defect with barbed sutures. We believe that compared with ordinary absorbable sutures, continuous suturing of the diaphragm with barbed sutures has the following advantages: less bleeding, absorbability, no knot response, tight sutures and no knots during the suture process, which greatly shortens the operation time. Moreover, re-strengthening the diaphragm reduces recurrence.

With the development of minimally invasive technology, thoracoscopy has gradually been used in the treatment of $\mathrm{CDH}$. We compared the effects of open surgery and thoracoscopy in the treatment of $\mathrm{CDH}$ in children. The operation time, postoperative mechanical ventilation time, postoperative hospital stay and postoperative CCU admission time in the thoracoscopy group were shorter than those in the open group. Therefore, thoracoscopic treatment of $\mathrm{CDH}$ has the advantages of less bleeding and faster recovery than traditional open surgery. However, the results of this analysis may be affected by selection bias. When the patient's condition is severe and the diaphragm defect is large, surgeons are more inclined to use open surgery to repair the defect.

This study compared the incidence of complications of thoracoscopy and open surgery. The literature reports that the most common complications of diaphragm hernia repair include intestinal obstruction, pneumothorax, pleural effusion and atelectasis. Other studies have reported scoliosis and pectus excavatum after open surgery [7]. In this study, the overall incidence of postoperative complications in the open group (45.16\%) was higher than that in the thoracoscopy group (36.17\%), which was equivalent to the incidence reported in the literature $[8,9]$. In this study, the main complications of the open group were lung infection, pleural effusion and respiratory failure. The main complications of the thoracoscopy group were respiratory failure and pneumothorax. The incidence of complications in the open group was higher than that in the thoracoscopy group, but there was no significant difference between the two groups ( $P>0.05$ ). There were 6 cases of pleural effusion and 7 cases of pneumonia in the open group. Open surgery has a larger traumatic area than thoracoscopic surgery and more exudation, which is likely to cause pleural effusion. Moreover, children are young and have poor resistance to lung infections. Two children in the open group died of respiratory failure after surgery. The possible causes were that the children had unstable haemodynamics before surgery, severe pulmonary dysplasia, poor cardiopulmonary function after surgery and could not tolerate open surgery.

This study compared the postoperative recurrence rate of the thoracoscopy and open groups. The recurrence rate of the thoracoscopy group (8.51\%) was higher than that of the open group (3.23\%), but the difference was not statistically significant. Some scholars have compared open surgery and thoracoscopic surgery, suggesting that the recurrence rate after thoracoscopic surgery for $\mathrm{CDH}$ is higher $[10,11]$ and that intraoperative hypercapnia and acidosis are more serious. The recurrence rate of thoracoscopic diaphragmatic hernia repair is reported to be between $0 \%$ and $25 \%$. Most authors have reported that the recurrence rate is higher than $15 \%[12,13]$. The recurrence rate in this study was lower than that reported in the literature, which may be attributed to the thoracoscopic diaphragmatic hernia repair being only performed in patients with a small diaphragm defect (defect diameter $<3 \mathrm{~cm}$ ). If a large 
defect was found under thoracoscopy, it was converted to open repair. In the open group, there were 2 cases of recurrence, which may have been due to poor development of the diaphragm, suture not reaching the edge of the normal diaphragm and a long-term increase in intra-abdominal pressure. The 2 cases of recurrence may have also been related to the large defect of the diaphragm, high tension of the diaphragm suture and loose suture. There were 4 cases of recurrence in the thoracoscopy group. We found that all recurrences occurred in the early stage of the use of thoracoscopy. The postoperative recurrence may have been related to several factors. (1) The surgical operation was performed under thoracoscopy, and the surgical field was enlarged. In addition, the surgeon could not accurately estimate the suture distance. As a result, the suture distance of each stitch was large, and the defect could not be closed well. (2) The diaphragm muscle tension was too large when sutured, and the sutured diaphragm muscle tissue was not thick enough, causing the diaphragm to tear. (3) The use of continuous sutures led to loose knots. In the later period, a purse-string suture with needled suture was used to repair the diaphragmatic defect, close the diaphragmatic hernia, restabilize and suture the edge of the defect with barbed sutures to achieve better clinical results. Scholars at home and abroad have also proposed the use of special equipment to assist in knotting and have achieved good results [14].

\section{Conclusions}

Thoracoscopic surgery and open surgery can effectively treat $\mathrm{CDH}$. Open surgery has advantages in patients with unstable haemodynamics, large diaphragm defects and abdominal malformations. Compared with conventional open surgery, thoracoscopy has the advantages of shorter operation time, less trauma, faster recovery and fewer complications, but there is a risk of recurrence. The choice of surgical method should be determined by the characteristics of diaphragmatic lesions and the experience of the clinician.

\section{List Of Abbreviations}

$\mathrm{CDH}$ (congenital diaphragmatic hernia), CCU (coronary care unit), CT (computed tomography)

\section{Declarations}

Ethics approval and consent to participate:The Medical Research Ethics Committee of Children's Hospital Affiliated to Chongqing Medical University approved the study, and this study obtained written informed consent from the families of all children.

Consent for publication: Not applicable.

Availability of data and materials: The datasets used and analyzed during the current study are available from the corresponding author upon reasonable request.

Competing interests: The authors declare that they have no competing interests. 
Funding: Not applicable.

Authors' contributions: ZSL analyzed and interpreted the patient data regarding congenital diaphragmatic eventration. JX was responsible for data collation. PZX, AY, and LYG were responsible for part of the design of the paper. PZX, AY, LYG, and WC performed for diaphragmatic hernia repair surgery.ZSL and WC were major contributors in writing the manuscript. All authors read and approved the final manuscript.

Acknowledgments: Not applicable.

\section{References}

[1] de Buys \oessingh AS®Dinh-Xuan AT囚Congenital diaphragmatic hernia: current status and review of

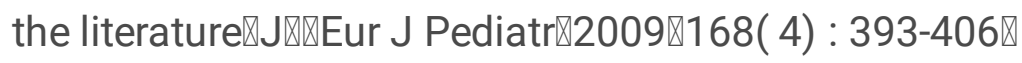

[2] Beresford MW, Shaw NJ. Outcome of congenital diaphragmatic hernia [J]. Pediatr Pulmonol, 2000, 30(3): 249-256.

[3] Brownlee EM, Howatson AG, Davis CF, et al. The hidden mortality of congenital diaphragmatic hernia: a 20-year review [J]. J Pediatr Surg, 2009, 44(2): 317-320.3

[4]Vijfhuize S $\triangle$ Deden AC $₫$ Costerus SA囚et al. Minimal access surgery for repair of congenital diaphragmatic hernia: is it advantageous? - An open review $\mathbb{J} \mathbb{U}$. Eur J Pediatr Surg,2012ख22( 5) : 364 373.

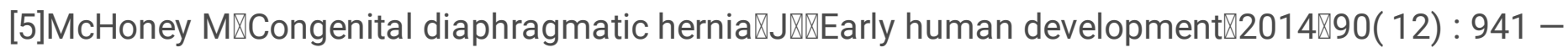
946区

[6]Stoll C『Alembik Y®Dott B囚et al.Associated malformations in cases with congenital diaphragmatic

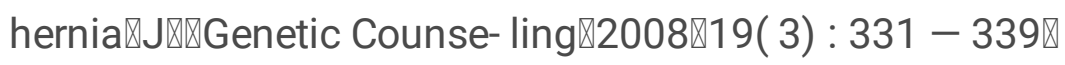

[7]Inoue M『Uchida KखOtake K囚et al. Thoracoscopic repair of congenital diaphragmatic hernia with countermeasures against reported complications for safe outcomes comparable to laparotomy $\mathbb{J} \mathbb{J}$. Surg Endosc $₫ 2016 \rrbracket 30$ ( 3 ) : $1014-1019$.

[8]Vijfhuize S, Deden AC, Costerus SA, Sloots CE, Wijnen RM. Minimal access surgery for repair of congenital diaphragmatic hernia: is it advantageous?: an open review. Eur J Pediatr Surg 2012;22:364-73.

[9]Gourlay DM, Cassidy LD, Sato TT, Lal DR, Arca MJ. Beyond feasibility: a comparison of newborns undergoing thoracoscopic and open repair of congenital diaphragmatic hernias. J Pediatr Surg 2009;44:1702-7.

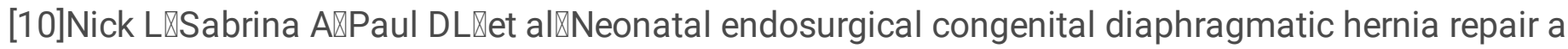

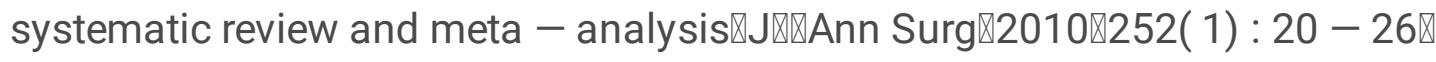


[11]Tsao K, Lally PA, Lally KP, The Congenital Diaphragmatic Hernia Study Group (2011) Minimally invasive repair of congenital diaphragmatic hernia. J Pediatr Surg 46:1158-1164

[12] McHoney M, Giacomello L, Nah SA, et al. Thoracoscopic repair of congenital diaphragmatic hernia: intraoperative ventilation and recurrence. J Pediatr Surg 2010;45:355-9.

[13] Lao OB, Crouthamel MR, Goldin AB, et al. Thoracoscopic repair of congenital diaphragmatic hernia in infancy. J Laparoendosc Adv Surg Tech 2010;20:271-6.

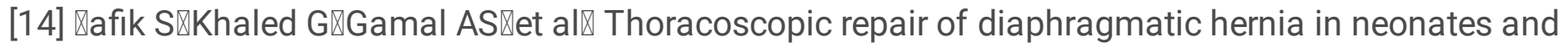

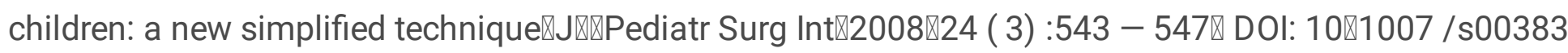
$-008 \bowtie 2128-6 \rrbracket$

\section{Tables}

Table 1.Comparison of general clinical data of the two groups of children $(n=109)$.

\begin{tabular}{|llll|}
\hline General information & $\begin{array}{l}\text { Open } \\
\text { group }(\mathrm{n}=61)\end{array}$ & $\begin{array}{l}\text { Thoracoscopy } \\
\text { group } \mathbb{n}=47 \rrbracket\end{array}$ & $\begin{array}{l}\mathrm{P} \\
\text { value }\end{array}$ \\
\hline Gender (male: female) & $36: 26$ & $25: 22$ & 0.612 \\
\hline Age of operation (months) & $8.83 \pm 6.11$ & $9.05 \pm 3.97$ & 0.755 \\
\hline Weight (kg) & $7.04 \pm 3.73$ & $6.55 \pm 2.44$ & 0.470 \\
\hline Location of diaphragmatic hernia (left: right) & $51: 11$ & $38: 9$ & 0.851 \\
\hline $\begin{array}{l}\text { Combined malformation (congenital heart disease: } \\
\text { congenital pulmonary dysplasia: others) }\end{array}$ & $29: 33: 10$ & $16: 20: 15$ & 0.106 \\
\hline
\end{tabular}

Table 2.Comparative analysis of operative-related indexes between the open group and thoracoscopy group $(n=109)$. 


\begin{tabular}{|c|c|c|c|}
\hline Operative related indexes & $\begin{array}{l}\text { Open } \\
\text { group }(n=61)\end{array}$ & $\begin{array}{l}\text { Thoracoscopy group } \\
\bigotimes n=47 \rrbracket\end{array}$ & $\begin{array}{l}P \\
\text { value }\end{array}$ \\
\hline Operation time $₫ \min \rrbracket$ & $103.66 \pm 36.25$ & $84.66 \pm 36.35$ & 0.000 \\
\hline Intraoperative blood loss $₫ \mathrm{ml} \rrbracket$ & $12.98 \pm 9.47$ & $7.71 \pm 6.11$ & 0.000 \\
\hline Total length of stay (days) & $22.75 \pm 11.84$ & $17.95 \pm 5.79$ & 0.000 \\
\hline $\begin{array}{l}\text { Intraoperative blood transfusion cases } \\
\text { (cases) }\end{array}$ & 4 & 5 & 0.432 \\
\hline Defect diameter $(<3 \mathrm{~cm}:>3 \mathrm{~cm})$ (cases) & $6: 56$ & $38: 9$ & 0.000 \\
\hline $\begin{array}{l}\text { Postoperative mechanical ventilation time } \\
\text { \days } \\
end{array}$ & $9.57 \pm 6.45$ & $5.09 \pm 2.74$ & 0.000 \\
\hline Postoperative hospital stay $\llbracket$ days $\rrbracket$ & $21.39 \pm 9.87$ & $13.53 \pm 4.23$ & 0.000 \\
\hline Postoperative CCU admission time $\rrbracket$ days $\rrbracket$ & $16.31 \pm 9.92$ & $6.21 \pm 3.20$ & 0.000 \\
\hline
\end{tabular}

Table 3.Comparative analysis of postoperative complications between the open group and thoracoscopy group $(n=109)$.

\begin{tabular}{|c|c|c|c|}
\hline Postoperative complications & Open group $(n=62)$ & Thoracoscopy group $\rrbracket n=47 \rrbracket$ & $P$ value \\
\hline Incision infection (cases) & प & 0 & 1.000 \\
\hline Incision dehiscence (cases) & 1 & 0 & 1.000 \\
\hline lung infection(cases) & 7 & 2 & 0.186 \\
\hline Respiratory failure(cases) & 5 & 7 & 0.259 \\
\hline Pleural effusion(cases) & 6 & 2 & 0.282 \\
\hline Atelectasis(cases) & 4 & 1 & 0.285 \\
\hline Chylothorax(cases) & 2 & 0 & 0.602 \\
\hline Pneumothorax(cases) & 2 & 5 & 0.118 \\
\hline Recurrence(cases) & 2 & 4 & 0.231 \\
\hline Mortality(cases) & 2 & 0 & 0.602 \\
\hline
\end{tabular}

\section{Figures}




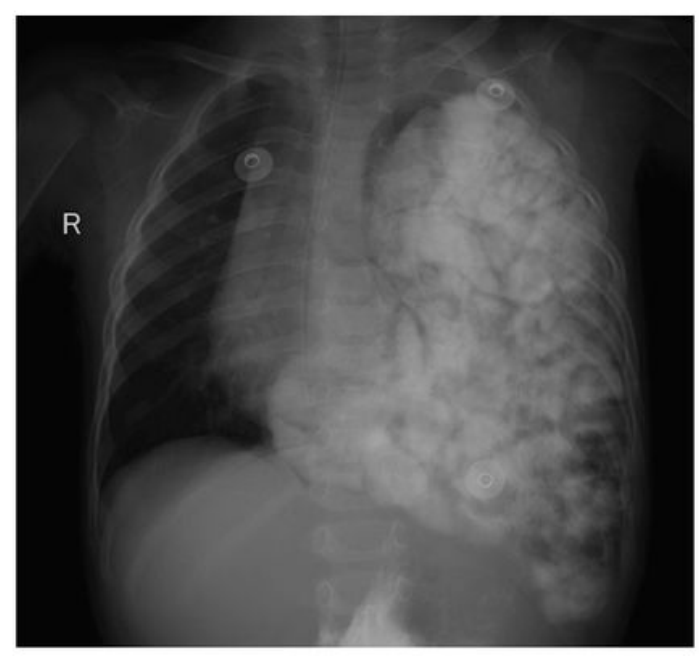

A

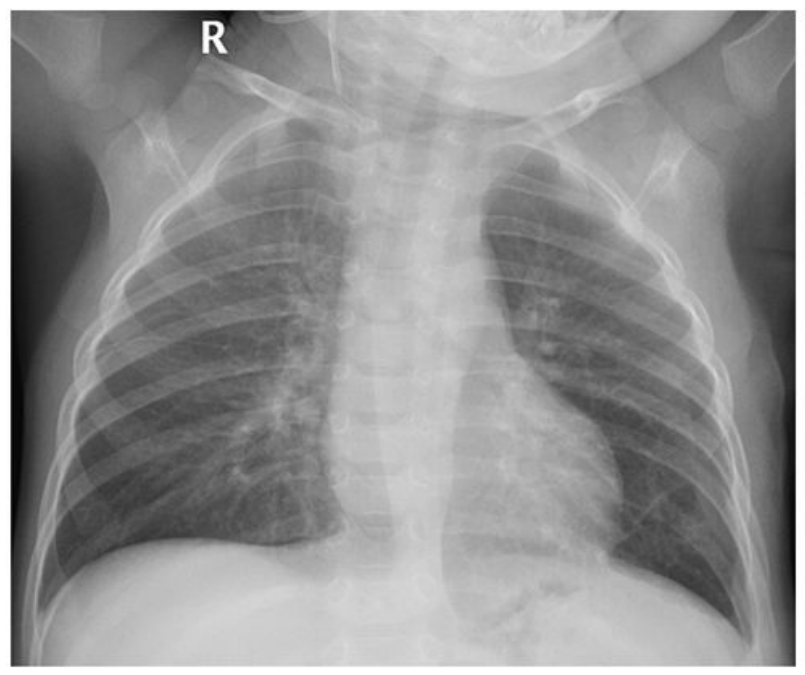

B

\section{Figure 1}

Comparison before and after diaphragmatic hernia repair. 